\title{
Hirschsprung's disease, Down syndrome, and missing heritability: too much collagen slows migration
}

\author{
Robert O. Heuckeroth \\ Children's Hospital of Philadelphia Research Institute and Perelman School of Medicine at the University of Pennsylvania, Philadelphia, Pennsylvania, USA.
}

\begin{abstract}
Hirschsprung's disease (HSCR) causes functional intestinal obstruction due to the absence of the enteric nervous system (ENS) in the distal bowel and is usually diagnosed shortly after birth or during childhood. While several genetic and nongenetic factors have been linked to HSCR, the underlying mechanisms that prevent ENS precursors from colonizing distal bowel during fetal development are not completely understood in many affected children. In this issue of the $J C l$, Soret and colleagues identify a new mechanism that causes HSCR-like disease in mice and involves deposition of excess collagen $\mathrm{VI}$ in the intestine by migrating ENS precursors as they colonize fetal bowel. Remarkably, their findings may explain some of the so-called missing heritability of HSCR and suggest a mechanism for increased HSCR incidence in children with Down syndrome (trisomy 21).
\end{abstract}

\section{Hirschsprung's disease:} congenital absence of distal bowel enteric nervous system

Hirschsprung's disease (HSCR) is a deadly birth defect in which the enteric nervous system (ENS) is missing from the end of the bowel $(1,2)$. Because the ENS controls most aspects of bowel function (3), even a short region of bowel without neurons and glia (i.e., aganglionosis) can be fatal. Over the past few decades many genetic and nongenetic causes of distal bowel aganglionosis have been identified, including mutations in RET, EDNRB, SOX1O, PHOX2B, and ZFHX1B (4, 5), but identifiable genetic problems do not explain HSCR occurrence in many affected children (6). Down syndrome also predisposes to HSCR.

The first definitive description of what is now called HSCR is attributed to Frederick Ruysch in 1691 (7), but the ancient Ayurvedic Sushruta Samhita describes "Baddha Gudodaram," a disorder remarkably like HSCR, as early as 2000 BCE (8). In 1886, Harald Hirschsprung reported two infants with the disorder that now bears his name $(9,10)$. The link between distal bowel aganglionosis and the massive proximal bowel distension that occurs with HSCR was not understood, however, until the pioneering studies of Swenson and Bill, Swenson et al., Bodian et al., Zuelzer and Wilson, and Whitehouse and Kernohan in 1948 and 1949, which showed that the absence of enteric neurons causes tonic contraction and functional obstruction (11-15). HSCR in a child with Down syndrome was first reported in 1956 (16), and increased occurrence of HSCR in individuals with Down syndrome was recognized in 1963 (17), suggesting that one or more genes on chromosome 21 contributes to HSCR etiology. Up to $10 \%$ of children with HSCR have Down syndrome, and $1 \%$ to $2 \%$ of children with Down syndrome have HSCR. Thus, Down syndrome increases HSCR risk about 50- to 100-fold above the general population risk $(21: 5,000)$ and is a common underlying partially penetrant cause of HSCR (4).

Over the past few decades there have been dramatic advances in our understanding of HSCR anatomy, embryology, physiology, and genetics $(4,5,18)$. Early studies by Yntema and Hammond showed that

Related Article: p. 4483

Conflict of interest: The author has declared that no conflict of interest exists.

Reference information: J Clin Invest. 2015;125(12):4323-4326. doi:10.1172/JCI85003.

the ENS forms from enteric neural crestderived cells (ENCDCs) that originate primarily in the vagal region of the neural tube (19). These ENCDCs proliferate vigorously as they colonize fetal bowel in a rostral to caudal progression, pursuing one of the longest migratory routes of any cell population during fetal development (Figure 1A). ENCDCs then differentiate into diverse neuron and glia subtypes that form a network in the bowel wall with about as many neurons as the spinal cord and every transmitter in the central nervous system $(3,18)$. Despite these advances in our understanding of ENS development, the causal link between trisomy 21 and increased HSCR incidence has remained elusive. Analysis of partial trisomy 21 phenotypes suggested that an extra copy of at least one gene in the interval from 33.5 to $46.25 \mathrm{Mb}$ increased HSCR risk (20). This interval contains 122 known genes, including DSCAM, BACE2, COL18A1, and COL6A1. A recent SNP association study suggested that excess DSCAM may be important for HSCR pathogenesis in Down syndrome, but excess DSCAM has not been confirmed experimentally to cause HSCR-like disease in model systems (21).

\section{Unbiased approach to identify genes critical for ENS development}

In this issue, Soret and colleagues took an unbiased, forward genetics approach and used insertional mutagenesis to identify potential regulators of neural crest-derived cell (NCC) migration (22). To simplify the screening process, Soret et al. randomly inserted a tyrosinase (Tyr) minigene, which rescues pigment production in NCCderived melanocytes, into albino FVB/N mice and then evaluated animals with nonuniform pigment patterns. Because melanocytes and the ENS are both neural crest derivatives, some mice with pigmentation defects were also expected to have ENS defects, mimicking the human "neurocristopathy" called Waardenburg-Shah syndrome (Waardenburg syndrome type 
A

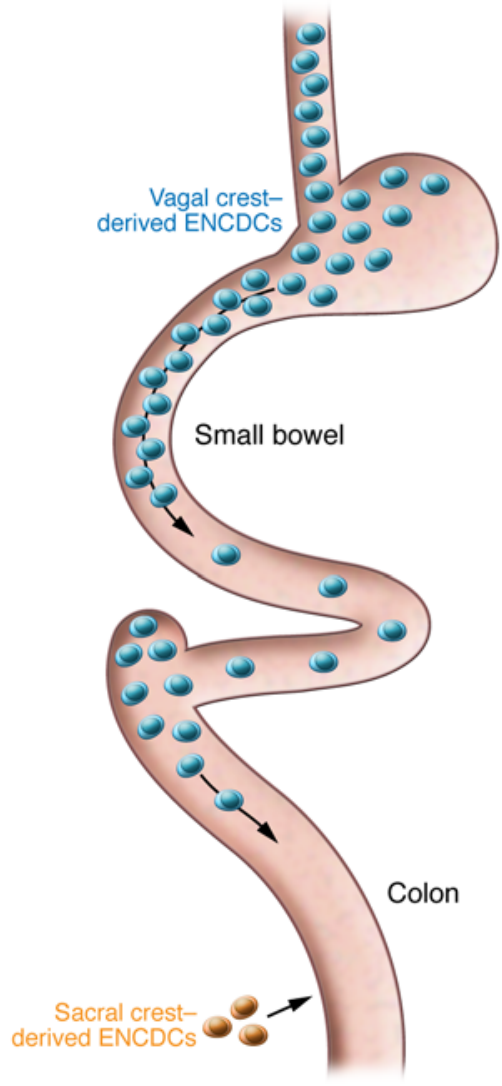

B

Nongenetic factors that affect ENS development

$\begin{array}{lll}\text { Nutrients } & \text { Medicines } & \text { Intestinal microbes } \\ \text { Vitamin A } & \text { Mycophenolate } & \text { Saccharomyces boulardii } \\ \text { Butyrate/resistant starch } & \text { Ibuprofen } & \text { Pediococcus acidilactici } \\ \text { n-3 polyunsaturated fatty acids } & \text { SSRIs } & \end{array}$

ENCDC-specific factors that influence ENS formation

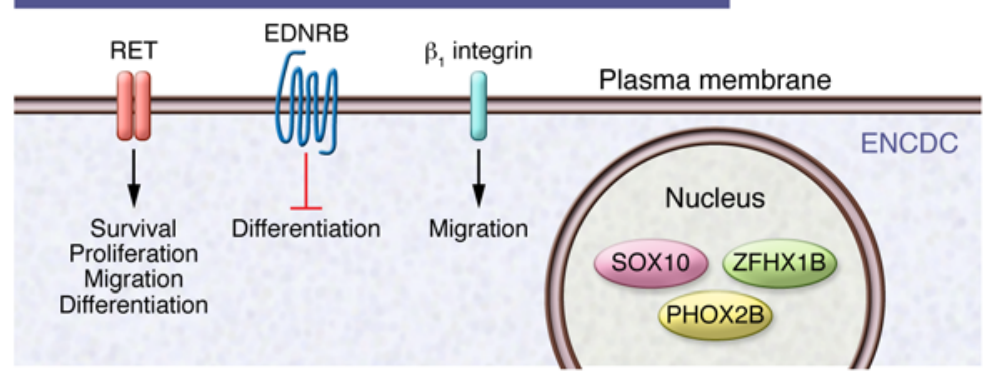

ENCDC-extrinsic factors that influence ENS formation

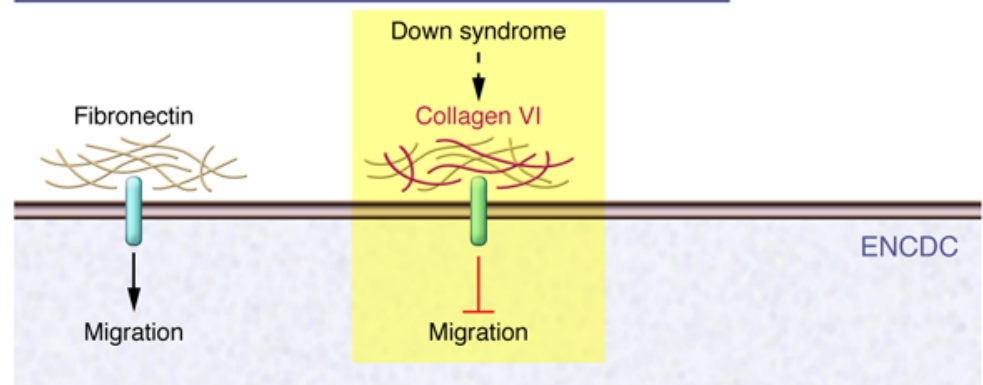

Figure 1. Formation of the ENS requires precise regulation of ENCDC proliferation, migration, and differentiation. (A) Schematic representation of the mouse bowel at E12.5. Vagal crest-derived ENCDCs (blue cells) migrate to and colonize the fetal bowel, where these ENS precursors differentiate into diverse neuron and glia subtypes. Sacral crest-derived ENCDCs also contribute to ENS formation in the hindgut, but to a lesser extent. HSCR is a life-threatening birth defect, characterized by a region of distal bowel in which the ENS is missing. (B) Simplified view of factors known to influence ENS development. Several genetic and nongenetic risk factors have been identified for this disease; however, the underlying cause of HSCR in many cases is not known. Nongenetic factors that cause HSCR-like disease in mice include maternal vitamin A deficiency and in utero exposure to mycophenolate. Several additional medicines, nutritional factors, and microorganisms also affect ENS development (27-29). HSCR-associated mutations have been identified in genes encoding factors that directly influence ENCDC behavior and phenotypes, including receptors RET and EDNRB, $\beta_{1}$ integrin, and transcription factors SOX10, PHOX2B, and ZFHX1B. Mutations of these factors result in dysfunctional ENS formation due to alterations in ENCDC survival, proliferation, migration, and/or other functions essential for normal innervation of the gut. Interactions between ENCDCs and the extracellular matrix along the migratory route also influence ENCDC behavior. In this issue, Soret and colleagues demonstrate that animals with excess collagen VI develop HSCR-like disease due to decreased ENCDC migration (22). Moreover, the inhibitory effect of collagen VI on ENCDC migration may partially explain why children with Down syndrome have an increased risk of HSCR, since collagen VI genes are on chromosome 21. For more details about ENS development and a more complete view of factors associated with HSCR, see refs. 4-6 and 18. SSRI, selective serotonin reuptake inhibitor.

4 [WS4]) that is characterized by a combination of skin or eye pigmentation defects, sensorineural hearing loss, and HSCR. Known causes of WS4 include inactivating mutations in EDNRB, EDN3, and SOX1O, but these gene defects account for only a small percentage of HSCR cases (5\% EDNRB , 4\% SOX10, <1\% EDN3), and most people with HSCR have normal pigmentation. Surprisingly, the insertional mutagenesis strategy used by Soret et al. led to increased expression of genes adjacent to the transgene insertion site and three new models of HSCR (Holstein $\left[\mathrm{Hol}^{\mathrm{Tg}}\right]$, ref. 22), TashT (23), and Spot (22). The genes identified by this approach would have been difficult to predict based on known ENS biology and would never have been discovered by traditional "knockout" approaches, because the affected genes are overexpressed in these models of HSCR and WS4, and the insertions involve noncoding regions of the genome.

Holstein homozygote $\left(\mathrm{Hol}^{\mathrm{Tg} / \mathrm{Tg}}\right)$ animals exhibit distal colon aganglionosis that is typical of $80 \%$ of human children with HSCR, but these animals also have quite extensive albinism, unlike typical human HSCR (22).
$\mathrm{Hol}^{T g / T g}$ mice have delayed colonization of fetal bowel by ENCDCs due to reduced cell migration, but there was no effect on the direction of ENCDC migration, rates of neuronal differentiation, proliferation, or cell death. In contrast, $\mathrm{Hol}^{\mathrm{Tg} / \mathrm{Tg}}$ mice had a smaller percentage of enteric glia-fated ENCDCs (SOX10 $\left.{ }^{+}, \mathrm{HuC} / \mathrm{D}^{-}, \mathrm{S} 100 \beta^{+}\right)$than WT mice at E15.5 and a larger percentage of undifferentiated ENCDCs (SOX10 ${ }^{+}$, $\left.\mathrm{HuC} / \mathrm{D}^{-}, \mathrm{S} 100 \beta^{-}\right)$, suggesting that the mutation slows glial differentiation, an effect unlikely to influence bowel colonization, which is complete by this time. 
The transgene insertion site in $\mathrm{Hol}^{\mathrm{Tg} / \mathrm{Tg}}$ mice is between collagen-6 $\alpha 4$ (Col6a4) and glycerate kinase (Glyctk) in a region that is syntenic to human chromosome 3q22. RNA sequencing (RNA-Seq) analysis on isolated E12.5 ENCDCs revealed that Col6a4 mRNA is markedly increased (about 250-fold) but that the mRNA levels of other nearby genes, including Glyctk, were not affected (22). The increase in Col6a4 mRNA was not detectable by RNA-Seq when the whole bowel was used instead of ENCDCs. This observation suggests that the transgene causes a cellautonomous increase in Col6a4 mRNA within migrating ENCDCs without affecting Col6a4 mRNA levels in other bowel cells. Consistent with these data, heterotopic grafting using WT and $\mathrm{Hol}^{T g / T g}$ tissues confirmed that $\mathrm{Hol}^{\mathrm{Tg} / \mathrm{Tg}}$ ENCDCs do not migrate efficiently into WT colons, moving at about half the speed of WT ENCDCs. Immunohistochemical staining confirmed increased collagen VI deposition around $\mathrm{Hol}^{\mathrm{Tg} / \mathrm{Tg}}$ ENCDCs as they colonize fetal bowel but no increase in collagen VI in regions of the bowel that had not been colonized by ENCDCs. Intriguingly, collagen VI protein levels in $\mathrm{Hol}^{\mathrm{Tg} / \mathrm{Tg}}$ bowels are only about 3-fold higher than those in WT bowels, an observation ascribed to the need to incorporate COL6A4 protein into trimeric collagen monomers that also contain COL6A1 and COL6A2, which are encoded by genes that are not overexpressed in $\mathrm{Hol}^{\mathrm{Tg} / \mathrm{Tg}}$ mice. Consistent with the hypothesis that excess collagen VI accounts for the HSCR-like phenotype, cultured WT ENCDCs migrated more slowly on collagen VI, and added collagen VI can reduced migration on fibronectincoated dishes (Figure 1B). One explanation for this observation is that collagen VI and fibronectin may compete for $\beta_{1}$ integrin binding since $\beta_{1}$ integrin is required for ENCDC migration (24). Alternatively, altered matrix stiffness or porosity might slow ENCDC migration and reduce glial differentiation.

\section{Increased collagen VI links HSCR and Down syndrome}

Soret et al. next hypothesized that their observations in mice may be relevant for human HSCR and could explain the increased HSCR occurrence in children with Down syndrome (22). The translation of observations made in Holstein mice to human biology required a substantial intuitive leap of faith. The human COL6A4 gene on chromosome 3 generates nonprocessed pseudogenes. In contrast, two of the genes encoding human collagen VI are on chromosome 21 (COL6A1 and COL6A2), and COL6A 1 is within a region defined as important for HSCR risk in human partial trisomy analyses (20). These collagen genes are expressed in ENCDCs and overexpressed in the skin of human fetuses with trisomy 21 , in which they probably exclusively interact with COL6A3 to form classical $\alpha 1-\alpha 2-\alpha 3$ trimeric collagen monomers. Soret and colleagues analyzed human colon muscle with myenteric ganglia from a cohort of patients with HSCR and healthy controls to test their hypothesis that excess collagen VI might increase human HSCR risk (22). Remarkably, collagen VI was more abundant in tissue surrounding myenteric ganglia of children with HSCR compared with that in control specimens (2-fold higher) and present at even higher levels surrounding myenteric ganglia of children with Down syndrome and HSCR (3-fold higher). Interestingly, there was no correlation between the age of the child and the level of collagen VI detected by immunohistochemistry.

\section{Conclusions and future directions}

Collectively, the studies by Soret and colleagues fit well with the hypothesis that some of the missing heritability in children with HSCR results from noncoding variants that alter gene expression (6) HSCR risk, however, remains challenging to define because so many genetic and nongenetic factors influence signaling pathways that control ENCDC survival, proliferation, differentiation, and migration (5), leading to complex gene-gene (25) and gene-environment interactions that affect ENS development $(18,26)$. The observation that elevated collagen VI levels reduce the speed of ENCDC migration fits well with long-standing observations that extracellular matrix molecules and matrix metalloproteinases influence ENS development (5). The hypothesis that increased collagen VI production by migrating ENCDCs in children with Down syndrome underlies increased HSCR risk is provocative and interesting. Studies to more formally test this hypothesis are challenging in humans, however, because HSCR is rarely diagnosed before birth. Moreover, ENCDCs migrate through the bowel during the first trimester of pregnancy, and only $1 \%$ to $2 \%$ of children with Down syndrome have HSCR. Nonetheless, this remarkable work provides new insight into ENS development, highlighting the need to understand the interaction of ENCDCs with the extracellular matrix, especially as we consider new cell-based therapies to treat enteric neuropathy.

\section{Acknowledgments}

Robert O. Heuckeroth was supported by the Irma and Norman Braman Endowment, the Suzi and Scott Lustgarten Center for GI Motility Endowment, the Children's Hospital of Philadelphia Research Institute, the Children's Discovery Institute of Washington University, St. Louis Children's Hospital (grants CH-II-1008-123 and CH-II-2010-390), the NIH (RO1 DK087715), and a Burroughs Wellcome Fund Clinical Scientist Award in Translational Research (grant 1008525).

Address correspondence to: Robert $\mathrm{O}$. Heuckeroth, Children's Hospital of Philadelphia Research Institute, 3615 Civic Center Boulevard, Abramson Research Center, Suite 1116i, Philadelphia, Pennsylvania 19104-4318, USA. Phone: 215.590.1209; E-mail: heuckerothr@email.chop.edu.

1. Heuckeroth RO. Hirschsprung disease. In: Faure C, DiLorenzo C, Thapar N eds. Pediatric Neurogastroenterology: Gastrointestinal Motility And Functional Disorders In Children. New York, New York, USA: Humana Press; 2013:271-283.

2. Skinner M. Hirschsprung's Disease. Curr Probl Surg. 1996;33(5):391-461.

3. Furness JB, Callaghan BP, Rivera LR, Cho HJ. The enteric nervous system and gastrointestinal innervation: integrated local and central control. Adv Exp Med Biol. 2014;817:39-71.

4. Amiel J, et al. Hirschsprung disease, associated syndromes and genetics: a review. JMed Genet. 2008;45(1):1-14.

5. Lake JI, Heuckeroth RO. Enteric nervous system development: migration, differentiation, and disease. Am J Physiol Gastrointest Liver Physiol. 2013;305(1):G1-G24.

6. Alves MM, et al. Contribution of rare and common variants determine complex diseases-Hirschsprung disease as a model. Dev Biol. 2013;382(1):320-329.

7. Leenders E, Sieber WK. Congenital megacolon observation by Frederick Ruysch - 1691 . J Pediatr Surg. 1970;5(1):1-3.

8. Raveenthiran V. Knowledge of ancient Hindu 
surgeons on Hirschsprung disease: evidence from Sushruta Samhita of circa 1200-600 BC. J Pediatr Surg. 2011;46(11):2204-2208.

9. Hirschsprung H. Stuhlträgheit Neugeborener in Folge von Dilatation und Hypertrophie des Colons. Jahrbuch für Kinderheilkunde und physische Erziehung (Berlin) 1888;27:1-7.

10. Skaba R. Historic milestones of Hirschsprung's disease (commemorating the 90th anniversary of Professor Harald Hirschsprung's death). J Pediatr Surg. 2007;42(1):249-251.

11. Swenson O, Bill AH Jr. Resection of rectum and rectosigmoid with preservation of the sphincter for benign spastic lesions producing megacolon; an experimental study. Surgery. 1948;24(2):212-220.

12. Swenson O, Rheinlander HF, Diamond I. Hirschsprung's disease; a new concept of the etiology; operative results in 34 patients. $N$ Engl J Med. 1949;241(15):551-556.

13. Bodian M, Stephens FD, Ward BC. Hirschsprung's disease and idiopathic megacolon. Lancet. 1949;1(6540):6-11.

14. Zuelzer WW, Wilson JL. Functional intestinal obstruction on a congenital neurogenic basis in infancy. Am J Dis Child. 1948;75(1):40-64.

15. Whitehouse FR, Kernohan JW. Myenteric plexus in congenital megacolon; study of 11 cases. Arch Intern Med (Chic). 1948;82(1):75-111.

16. Borbolla Vacher L, Garcia Palacio A, Martinez Garcia W. Hirschsprung's disease in a 38-day mon- goloid. Rev Cubana Pediatr. 1956;28(8):473-484.

17. Bodian M, Carter OO. A family study of Hirschsprung's disease. Ann Hum Genet. 1963;26(3):261-277.

18. Avetisyan M, Schill EM, Heuckeroth RO. Building a second brain in the bowel. JClin Invest. 2015;125(3):899-907.

19. Yntema CL, Hammond WS. The origin of intrinsic ganglia of trunk viscera from vagal neural crest in the chick embryo. JComp Neurol. 1954;101(2):515-541.

20. Korbel JO, et al. The genetic architecture of Down syndrome phenotypes revealed by high-resolution analysis of human segmental trisomies. Proc Natl Acad Sci US A. 2009;106(29):12031-12036.

21. Jannot AS, et al. Chromosome 21 scan in Down syndrome reveals DSCAM as a predisposing locus in Hirschsprung disease. PLoS One. 2013;8(5):e62519.

22. Soret R, et al. A collagen VI-dependent pathogenic mechanism for Hirschsprung's disease. J Clin Invest. 2015;125(12):4483-4496.

23. Bergeron $\mathrm{KF}$, et al. Male-biased aganglionic megacolon in the TashT mouse line due to perturbation of silencer elements in a large gene desert of chromosome 10. PLoS Genet. 2015;11(3):e1005093.

24. Breau MA, Dahmani A, Broders-Bondon F, Thiery JP, Dufour S. $\beta 1$ Integrins are required for the invasion of the caecum and proximal hindgut by enteric neural crest cells. Development. 2009;136(16):2791-2801.

25. McCallion AS, Stames E, Conlon RA, Chakravarti A. Phenotype variation in two-locus mouse models of Hirschsprung disease: tissue-specific interaction between Ret and Ednrb. Proc Natl Acad Sci U S A. 2003;100(4):1826-1831.

26. Lake JI, Tusheva OA, Graham BL, Heuckeroth RO. Hirschsprung-like disease is exacerbated by reduced de novo GMP synthesis. JClin Invest. 2013;123(11):4875-4887.

27. Saurman V, Stevanovic K, Israelyan N, Li ZS, Gershon M, Snyder I, Margolis KG. Selective serotonin reuptake inhibitors have critical and long-lasting effects on enteric nervous system development and gastrointestinal function. Paper presented at: North American Society for Pediatric Gastroenterology, Hepatology and Nutrition Annual Meeting; October 7-11, 2015; Washington DC.

28. Kamm K, Hoppe S, Breves G, Schroder G, Schemann M. Effects of the probiotic yeast Saccharomyces boulardii on the neurochemistry of myenteric neurons in pig jejunum. Neurogastroenterol Motil. 2004;16(1):53-60.

29. Di Giancamillo A, Vitari F, Bosi G, Savoini G, Domeneghini C. The chemical code of porcine enteric neurons and the number of enteric glial cells are altered by dietary probiotics. Neurogastroenterol Motil. 2010;22(9):e271-e278. 